\title{
Concepción de aprendizaje, estrategias metacognitivas y experticia disciplinar en estudiantes universitarios de psicología
}

\section{Learning conception, metacognitive strategies and disciplinary expertise in psychology undergraduate students}

\author{
José Sandoval Díaz ${ }^{1}$ \\ Universidad de Chile, Chile \\ (Rec: septiembre de 2015 - Acept: noviembre de 2015)
}

\begin{abstract}
Resumen
El objetivo de este trabajo es determinar la(s) influencia(s) de los modelos de nivel de pericia y de metacognición, sobre la concepción de aprendizaje en estudiantes universitarios de psicología. Participaron 184 sujetos de ambos sexos, pertenecientes a la ciudad de Arica, Chile. Se utilizó el Inventario de Concepciones de Aprendizaje (Martínez, 2000) y el Cuestionario de Estrategias Metacognitivas (O’Neil \& Abedi, 1996). Los resultados apuntan a que el nivel de pericia y las estrategias metacognitivas correlacionan significativamente con la concepción de aprendizaje. Sin embargo, en un análisis detallado para cada tipo de concepción de aprendizaje se encontraron diferencias estadísticas significativas entre ambos modelos explicativos. Estos resultados evidencian que las concepciones interpretativa y constructiva se ven mayormente influenciadas por el uso de estrategias metacognitivas de aprendizaje, en contraposición al tipo directivo, el cual es explicado, en mayor medida, por el nivel de pericia de los estudiantes.
\end{abstract}

Palabras clave: concepciones de aprendizaje, estrategias metacognitivas, nivel de pericia en psicología.

\begin{abstract}
The aim of this work is to determine how expertise level models and metacognition influence on undergraduate psychologist learning conception. 144 male and female participants from the city of Arica in Chile took part in this study. They responded the learning conception inventory (Martínez, 2000) and the metacognitive strategy questionnaire (O’Neil \& Abedi, 1996). Results showed that expertise levels and metacognitive strategies significantly correlated with learning conceptions. However, in a detailed analysis design for each type of learning, statistically significant differences were found between the two explanatory models. These results suggest that the constructive and interpretative conceptions are largely influenced by the use of specific metacognitive strategies, in spite of the directive strategy, which is mainly explained by the student's expertise levels.
\end{abstract}

Keywords: learning conceptions, metacognitive strategies, expertise level in psychology.

\footnotetext{
Correspondencia a: José Sandoval Díaz. Escuela de psicología, Universidad de Tarapacá, 18 de Septiembre 2222, Arica. E-mail:josesandovald @ ug.uchile.cl
} 


\section{Introducción}

En las últimas décadas, tanto en psicología como en educación, la investigación sobre el papel y las características del proceso de aprendizaje ha sido realizada desde múltiples y variados enfoques. Uno de estos enfoques ha sido el del cambio conceptual, el cual ha logrado una amplia cabida en el campo psicoeducativo. Este enfoque se centra en la construcción psicológica de conceptos (en adelante concepciones), abarcando nociones del campo de las ciencias naturales tales como energía y gravedad, pero también de las ciencias sociales, como por ejemplo justicia, moral y aprendizaje, por mencionar sólo algunas. Para este enfoque, las concepciones se articulan y organizan en forma de teorías personales, las cuales pueden experimentar un proceso de cambio por enriquecimiento, reelaboración, revisión o reestructuración (Carey, 1985a; 1985b). A su vez, se ocupa de concepciones de carácter intuitivo, examinando, posteriormente, su proceso de variación o restructuración conceptual (Dysktra, Boyle \& Monarch, 1992; Hewson \& Thorley, 1989; Kuhn \& Lao, 1998; Limón, 2001; Rodríguez Moneo \& Carretero, 1996; White \& Gunstone, 1989).

En el campo aplicado de la educación formal se sostiene que detrás de las acciones y/o estrategias empleadas por los estudiantes existiría un cuerpo teórico fundamentado por un conjunto de supuestos coherentes o inconexos entre sí, al cual se ha denominado concepción de aprendizaje (Säljö, 1979). Este conjunto de supuestos establecería cierta direccionalidad para el alcance de los objetivos de aprendizaje propuestos por el estudiante, resaltando el papel conceptual de las creencias sobre el aprendizaje (Pintrich, Marx \& Boyle, 1993). En cuanto al proceso de desarrollo de la concepción, en su génesis, se concibe en términos de reproducción o copia fiel del objeto o del proceso a adquirir. Sin embargo, esto podría modificarse adquiriendo gradualmente una mayor elaboración y/o estructuración, mediante la acción constructiva del sujeto, la relatividad de su pensamiento y el potencial de cambio cognitivo de las ideas iniciales.

En cuanto a los tipos de concepción de aprendizaje, Pozo y Scheuer (1999) identifican tres: la directiva, la interpretativa y la constructivista. La concepción directiva considera que existe una correspondencia directa entre ciertas condiciones y el resultado del aprendizaje, partiendo del supuesto que el conocimiento debiese reproducir fielmente la realidad externa. Por otro lado, la concepción interpretativa plantea que la actividad personal del estudiante es imprescindible para lograr un óptimo aprendizaje, siendo esta actividad crucial en la mediación entre condiciones y resultados del aprendizaje. Por último, la concepción constructivista asume que el aprendizaje implicaría procesos (re) constructivos, los cuales producen nuevas relaciones y conocimientos, atribuyendo de esta manera a los procesos mediacionales la función transformadora y de reelaboración del objeto aprendido. Esta concepción implicaría, a su vez, una clara disposición al cambio, así como la activación de mecanismos que facilitan el enriquecimiento y/o reestructuración del conocimiento (Tynjälä, 1997; 1999).

En el ámbito de la investigación empírica aplicada al contexto de educación universitaria, los resultados obtenidos han sido diversos y hasta contradictorios. Por un lado, si bien se ha encontrado presencia de concepciones de aprendizaje de mayor elaboración, del tipo interpretativo y constructivista, en estudiantes de este nivel (Martínez, 2000), parece no ser exclusiva de esta población, encontrando evidencia también en estudiantes de enseñanza primaria y secundaria (Pozo $\&$ Scheuer, 1999). A su vez, de modo contra intuitivo, se ha encontrado presencia de concepciones de aprendizaje de menor elaboración, es decir del tipo directivo, en estudiantes universitarios (Máiquez, Rodrigo, Capote $\&$ Vermaes, 2000) e incluso en personas dedicadas a la enseñanza (Pozo \& De la Cruz, 2003).

Debido a la diversidad de resultados, han surgido una serie de investigaciones que buscan identificar e indagar posibles variables mediadoras en el desarrollo de la concepción de aprendizaje universitario. A partir de la investigación desarrollada por Martínez (2004) en torno al desarrollo de la concepción de aprendizaje en 276 estudiantes españoles pertenecientes a la carrera de psicología, el autor identifica la influencia de dos variables en el desarrollo del aprendizaje: el nivel de pericia o conocimiento de dominio específico, por un lado, y las estrategias metacognitivas de aprendizaje por el otro. Ambas variables se describen a continuación.

\section{Nivel de pericia}

La investigación sobre nivel de pericia se ocupa de ámbitos específicos del conocimiento, instalando así el término de dominio específico, el cual, siguiendo a Reif y Larkin (1991), se define como: "la colección de conocimiento declarativo y procedimental necesario para atender a metas particulares, lo que implica el uso de conceptos específicos, las relaciones entre ellos y los métodos empleados en el mismo" (p. 735). Por su parte, Pozo (2003) hace referencia a los dominios 
específicos como "un conjunto de sucesos o contextos que procesamos de acuerdo con las mismas restricciones o principios" (p. 201). Así pues, desde este enfoque $y$, particularmente, en el dominio específico de las disciplinas académicas, se postula que a medida que aumenta el grado de experticia en cierto dominio, también se enriquece la profundidad de la concepción de aprendizajes de base, siendo posible identificar niveles más complejos de elaboración y de aplicación de estrategias de aprendizaje. En este sentido, remitirnos a la experticia conlleva diferencias en la estructura de conocimiento de los sujetos, en lo referente a su avance y desarrollo de novatos a expertos en el área en cuestión (Glaser, 1982). En tanto a la evidencia obtenida entre nivel de pericia y concepción de aprendizaje universitario, ésta muestra que a lo largo del proceso de formación existirían diferencias significativas entre las creencias epistemológicas sobre el aprendizaje, dependiendo del tipo y avance educativo que presente el estudiante (Schommer, 1993; Schommer, Calvert, Gariglietti \& Bajaj, 1997). Estos resultados convergen con investigaciones realizadas en estudiantes universitarios de distintas disciplinas científicas (Alexander, Murphy, Guan \& Murphy, 1998; Boulton-Lewis, Marton, Lewis \& Wilss, 2000; Boulton-Lewis, Smith, McCrindle, Burnett \& Campbell, 2001). En síntesis, esto parece apoyar la hipótesis de restructuración en la concepción de aprendizaje debido al aumento de la experticia adquirida a lo largo de los años de formación universitaria. Sin embargo, aún no quedaría claro cuáles serían los pasos o situaciones que generan dichos cambios, así como cuáles serían los factores asociados a este cambio (Lonka, Joram \& Bryson, 1996).

\section{Estrategias metacognitivas de aprendizaje}

La segunda variable identificada por Martínez (2004) como responsable de la profundización de la concepción de aprendizaje corresponde a las estrategias metacognitivas. Desde el campo educativo y la psicología cognitiva, se ha señalado a la metacognición como el proceso que permite organizar adecuadamente la información, facilitando así la atención, motivación, aprendizaje, recuerdo, comprensión y control de los procesos cognitivos. Por tanto, se asume que la metacognición es el proceso base para las tareas intelectuales generales (Danserau, 1978; DeCharms, 1972; Dweck, 1975; Mischel \& Baker, 1975; Nisbet \& Shucksmith, 1987; Valle et al., 1999; Vermunt, 1996; 1998). Es Flavell (1979) quien en la década de los setenta del siglo pasado acuña el término, definiéndolo como la facultad de conocer el propio conocimiento. Una década después, se replantea la definición del concepto, identificando y distinguiendo dos componentes centrales:

a) El conocimiento sobre los procesos cognitivos (saber qué), relativo a personas, estrategias o tareas.

b) La regulación de los procesos cognitivos (saber cómo), relacionada con la planificación, el control y evaluación de los procesos cognitivos (Brown, Bransford, Ferrara \& Campione, 1983).

A partir de este segundo componente, se enfatizó el rol procedimental de la planificación cognitiva, es decir, de las actividades que permiten regular, dirigir y controlar los procesos cognitivos. Bajo este ámbito procedimental, y de acuerdo con Schraw y Moshman (1995), se distinguen tres tipos de estrategias metacognitivas de aprendizaje: la planificación, el control y la evaluación. La planificación incluye la selección de estrategias previas a la ejecución, así como la selección de recursos adecuados para realizar la tarea. Así, por ejemplo, tenemos la secuenciación de actividades y asignación de tiempo requerido. El control remite a la revisión que se realiza durante la tarea o autoevaluación durante la ejecución. Un ejemplo de ello es la consideración del cómo se está ejecutando la tarea o variación de las estrategias que no están dando resultado. Y por último, la evaluación comprende la consideración de los productos o resultados de la ejecución y una valoración general. Este subproceso se define como la valoración de lo realizado y lo que ha quedado por mejorar de la actividad total (Schraw \& Moshman, 1995).

En lo empírico, estudios sobre metacognición en estudiantes universitarios (Boekaerts, 1999; Boekaerts \& Niemivirta, 2000) concluyen que la activación de la facultad de autorregulación implicaría la utilización de estrategias metacognitivas, facilitando así, procesos de mayor elaboración y restructuración del aprendizaje. Sin embargo, el uso y desempeño de estas estrategias sería sensible a las características del contexto educativo. Por ejemplo, un contexto educativo tradicional, es decir aquel centra su mirada en el docente y la enseñanza, presentaría una menor activación y uso de este tipo de estrategias por parte del estudiantado.

Por otro lado, en torno a la relación entre nivel de pericia y estrategias metacognitivas, Nickerson, Perkins y Smith (1985) plantean que estudiantes expertos no sólo presentan mayor nivel de aprendizaje que estudiantes novatos, sino que "saben que saben más", empleando mejor el conocimiento en términos de organización, accesibilidad y adquisición (Echeverría, Mateos, Pozo $\&$ Scheuer, 2001). A su vez, algunas investigaciones 
han considerado que la falta de conocimiento de dominio específico sería una de las principales razones por la cual estudiantes novatos no serían metacognitivamente más hábiles que los expertos, presentando así un conocimiento fragmentado y menos comprensivo (Martínez, 1999; 2004).

Teniendo en cuenta estos antecedentes, el presente trabajo tiene por objeto determinar el grado de influencia del nivel de pericia en psicología y de las estrategias metacognitivas sobre el tipo de concepción de aprendizaje en estudiantes universitarios chilenos, enmarcándolos, al igual que el estudio de Martínez (2004), en el dominio específico de la psicología. En consecuencia, la originalidad de este trabajo reside en identificar el tipo y grado de influencia de ambas variables sobre el desarrollo de las concepciones de aprendizaje en estudiantes universitarios chilenos. Por lo tanto, se busca contrastar las siguientes hipótesis: (1) el nivel de pericia en psicología se asocia e influye sobre el tipo de concepción de aprendizaje, (2) las estrategias metacognitivas se asocian e influyen sobre el tipo de concepción de aprendizaje y (3) ambas variables se asocian e influyen sobre el tipo de concepción de aprendizaje.

Bajo esta propuesta de análisis, Brew (2001) ha planteado la necesidad de realizar mayor investigación en torno al enfoque del cambio conceptual, así como también en el posible establecimiento de relaciones explicativas entre esta investigación y el uso de estrategias de enseñanza, ampliando así el dominio explicativo sobre el aprendizaje universitario y sus condiciones.

\section{Metodología}

\section{Participantes}

Se utilizó un muestreo no probabilístico al seleccionar, en primer lugar, las dos únicas universidades que imparten la carrera de psicología en la ciudad de Arica, y en segundo lugar, al seleccionar sólo aquellos estudiantes que cumplieran con los criterios de inclusión muestral establecidos de acuerdo al nivel de pericia. Participaron en el estudio un total de 184 estudiantes universitarios pertenecientes a la carrera de psicología. Estos se distribuyeron según el nivel de pericia que presentaban (Baja=84; Media=59 y Alta=41). Los participantes cursaban el primer semestre (pericia baja), quinto semestre (pericia media), y noveno semestre (pericia alta) del año académico de las facultades de psicología de la Universidad de Tarapacá y Universidad Santo Tomás. La edad promedio de los(as) estudiantes era de 22,42 años con una desviación estándar de 4,82. Con respecto al sexo de los participantes, 59 eran hombres (el 32,1\%), mientras que 125 eran mujeres (el 67,9\%).

\section{Instrumentos y variables}

Los instrumentos y variables operacionalizadas fueron los siguientes:

- CONAPRE: Inventario de auto-reporte que evalúa la concepción de aprendizaje. Instrumento diseñado y validado en el año 2000 por Martínez Fernández. El cuestionario consta de 15 ítems y, aunque inspirado inicialmente en la categorización de Säljö (1979), fue reformulado más tarde, tras un análisis factorial, en tres factores que corresponden a cada una de las categorías definidas por Pozo y Scheuer (1999) para la concepción de aprendizaje: directiva, interpretativa y constructivista.El formato de respuesta de la encuesta debe ser valorado en una escala que va desde uno (nunca) a cinco (siempre). Con los puntajes obtenidos se realizó el análisis de fiabilidad de escala tanto por factor como por escala completa. Respecto a las confiabilidades por factor, se obtuvo un alpha de Cronbach de 0.73 para el factor directivo, de 0.59 para el factor interpretativo y de 0.77 para el factor constructivista. Mientras que a nivel general, la confiabilidad obtenida fue de 0.71 .

- Inventario sobre estrategias metacognitivas de aprendizaje: Se utilizó el cuestionario de autoreporte de O'Neil y Abedi (1996). El instrumento se divide en dos factores: Planificación y Evaluación-Control. Este instrumento posee veinte reactivos que se contestan de acuerdo a una escala que va desde uno (nunca) a cinco (siempre). Con los puntajes obtenidos se realizó el análisis de fiabilidad de escala tanto por factor como por el instrumento completo. Respecto a las confiabilidades por factor, se obtuvo un alpha de Cronbach de 0.76 para el factor planificación y de 0.82 para el factor control evaluación. Mientras que a nivel general, la confiabilidad obtenida fue de 0.80 (Alpha de Cronbach).

- Nivel de pericia: Esta variable se operacionalizó en función del semestre que cursaba el estudiante. La carrera de psicología, en ambas universidades, contempla un total mínimo de 11 semestres. En el momento de la realización del estudio, correspondiente al primer semestre del año académico, se seleccionaron 84 estudiantes que se encontraban cursando el primer 
semestre $(45,7 \%)$, los cuales fueron codificados como nivel de pericia bajo; 59 estudiantes se encontraban cursando el quinto semestre $(32,1 \%)$, los cuales fueron codificados como nivel de pericia medio, y por último, 41 estudiantes se encontraban cursando el noveno semestre $(22,3 \%)$, los cuales fueron codificados como nivel de pericia alto.

\section{Procedimiento}

En primer lugar, se identificaron y seleccionaron las dos universidades que impartían la carrera de psicología en la ciudad de Arica. Tras la obtención de los permisos correspondientes con ambas direcciones de escuela, se estableció una reunión informativa con los profesores de las asignaturas con mayor cantidad de estudiantes inscritos de primer, quinto y noveno semestre respectivamente. En tanto a la producción de datos, se entregó a cada uno de los estudiantes cuadernillos con los instrumentos descritos, consignado el nivel cursado y antecedentes sociodemográficos generales. El tiempo de respuesta de los cuadernillos fue de 15 minutos. Tras la aplicación del instrumento, se procedió a la digitación de los datos y su posterior análisis, utilizando el programa IBM SPSS Statistics 18. Se efectuaron análisis de correlación de Pearson, análisis de varianza (ANOVA) con prueba de comparaciones múltiples y análisis de regresión múltiple con el método de extracción de pasos sucesivos. Respecto a la dimensión ética de la investigación, con la finalidad de resguardar la confidencialidad de la información obtenida, el cuadernillo de cuestionarios fue respondido de forma voluntaria, anónima y supervisada por el investigador responsable. Por último, la investigación no contó con financiamiento externo.

\section{Resultados}

\section{Asociaciones entre las variables centrales del estudio}

Como primer objetivo se indagó en el grado de asociación entre los puntajes obtenidos en estrategias metacognitivas y los tres tipos de concepción de aprendizaje por medio del coeficiente de correlación lineal de Pearson. De acuerdo a lo observado (Ver tabla 1), la variable estrategias metacognitivas presenta una relación negativa y estadísticamente significativa con la dimensión directiva $r(184)=-0,15, p=0,01$. En cambio, mostró una relación positiva y significativa con la dimensión interpretativa $r(184)=0.61, p=0.01$; y la dimensión constructiva $r(184)=0.66, p<.01$.

Tabla 1

Correlación entre estrategias metacognitivas y tipos de concepción de aprendizaje

\begin{tabular}{|c|c|c|c|c|}
\hline Variables & $\begin{array}{c}\text { Estrategias } \\
\text { Metacognitivas }\end{array}$ & Concepción directiva & $\begin{array}{c}\text { Concepción } \\
\text { interpretativa }\end{array}$ & $\begin{array}{c}\text { Concepción } \\
\text { constructivista }\end{array}$ \\
\hline $\begin{array}{c}\text { Estrategias } \\
\text { metacognitivas }\end{array}$ & & $-0,15$ & 0,60 & 0,66 \\
\hline
\end{tabular}

$\mathrm{p}<0,001$

\section{Análisis de varianza de diferencias de medias} (ANOVA)

Como segundo objetivo, se analizó la existencia de diferencias significativas entre los tres tipos de concepción de aprendizaje (directiva, interpretativa y constructivista) en función de los tres niveles de pericia (bajo, medio y alto). Para esto se utilizó el análisis de varianza ANOVA de diferencias de medias sobre los tres tipos de concepción de aprendizaje. Para la concepción directiva (Ver tabla 2) se obtuvo una diferencia estadísticamente significativa ante nivel de pericia, $\mathrm{F}(2,181)=48,071, \mathrm{p}<0.00$. Para determinar que medias son las que difieren, se utilizó la prueba post hoc de Bonferroni, la cual mostró que hay diferencias estadísticamente significativas entre el nivel de pericia bajo, con pericia media $(\mathrm{p}<0.00)$ y alta $(\mathrm{p}<0.00)$. 
Tabla 2

ANOVA de un factor para concepción de aprendizaje directivo por niveles de pericia y prueba de Bonferroni entre niveles de pericia baja, media y alta

\begin{tabular}{cccccc}
\hline Directiva & N & Media & DE & Df & F \\
\hline Baja & 84 & 15.29 & 2.115 & $2 / 181$ & $48.071^{*}$ \\
Media & 59 & 11.86 & 3.065 & & \\
Alta & 41 & 10.73 & 3.317 & & \\
Pericia Baja & Sig. & Pericia Media & Sig. & Pericia Alta & Sig. \\
Media & $0.000^{*}$ & Baja & $0.000^{*}$ & Baja & $0.000^{*}$ \\
Alta & $0.000^{*}$ & Alta & 0.130 & Media & 0.130 \\
\hline
\end{tabular}

$* \mathrm{p}<0.05$.

Para la concepción interpretativa (Ver tabla 3) también se obtuvo una diferencia estadísticamente significativa ante el nivel de pericia, $\mathrm{F}(2,181)=10,195, \mathrm{p}<$
0.00 . De acuerdo a la prueba post hoc, existen diferencias estadísticamente significativas entre el nivel de pericia bajo con pericia media $(\mathrm{p}<0.00)$ y alta $(\mathrm{p}<0.00)$.

Tabla 3

ANOVA de un factor para concepción de aprendizaje interpretativo por niveles de pericia y prueba de Bonferroni entre niveles de pericia baja, media y alta

\begin{tabular}{|c|c|c|c|c|c|}
\hline Directiva & $\mathrm{N}$ & Media & DE & Df & $\mathrm{F}$ \\
\hline Baja & 84 & 20.01 & 4.085 & $2 / 181$ & $10.195 *$ \\
\hline Media & 59 & 22.59 & 3.024 & & \\
\hline Alta & 41 & 22.20 & 3.494 & & \\
\hline Pericia Baja & Sig. & Pericia Media & Sig. & Pericia Alta & Sig. \\
\hline Media & $0.000^{*}$ & Baja & $0.000 *$ & Baja & $0.006 *$ \\
\hline Baja & $0.006^{*}$ & Alta & 1 & Media & 1 \\
\hline
\end{tabular}

$* \mathrm{p}<0.05$.

Por último, en cuanto a la concepción constructiva (ver tabla 4), a su vez, también se obtuvo diferencias estadísticas frente a nivel de pericia, $\mathrm{F}(2,181)=$
$17,146, \mathrm{p}<0.00$. De acuerdo a la prueba post hoc, se encontraron sólo diferencias significativas entre el nivel de pericia baja y alta $(\mathrm{p}<0.00)$.

Tabla 4

ANOVA de un factor para concepción de aprendizaje constructivo por niveles de pericia y prueba de Bonferroni entre niveles de pericia baja, media y alta

\begin{tabular}{cccccc}
\hline Directiva & $\mathrm{N}$ & Media & DE & Df & F \\
\hline Baja & 84 & 15.67 & 3.428 & $2 / 181$ & $17.146^{*}$ \\
Media & 59 & 16.15 & 3.666 & & \\
Alta & 41 & 19.63 & 3.039 & Pericia Alta & Sig. \\
Pericia Baja & Sig. & Pericia Media & Sig. & Baja & $0.000^{*}$ \\
Media & 1 & Baja & Media & $0.000^{*}$ \\
Baja & $0.000^{*}$ & Alta & $0.000^{*}$ & & \\
\hline
\end{tabular}

$* \mathrm{p}<0.05$. 


\section{Análisis de Regresión}

Como último objetivo, para determinar el grado de influencia de ambas variables predictoras: a) nivel de pericia y b) estrategias metacognitivas sobre cada tipo de concepción de aprendizaje, se utilizó el análisis de regresión múltiple con el método de extracción de pasos sucesivos (stepwise), esto con el fin de que sólo ingresasen, en orden de importancia, las variables significativas estadísticamente. La tabla 5 presenta una síntesis de los modelos evaluados

Tabla 5

Análisis de regresiones con procedimiento de pasos sucesivos para cada tipo de concepción de aprendizaje

Concepción de aprendizaje directivo

\begin{tabular}{cccc}
\hline Modelo & F & Beta & p \\
\hline Pericia & $F(1,181)=46,05 p=, 00$ &,- 63 &, 00 \\
$\begin{array}{c}\text { Estrategia metacognitiva } \\
\text { planificación }\end{array}$ &, 13 &, 04 \\
\hline R2 $=, 34 \quad$ R2 Ajustado $=, 33$ & &
\end{tabular}

Concepción de aprendizaje interpretativo

\begin{tabular}{cccc}
\hline Modelo & $F$ & Beta & $p$ \\
\hline Estrategias Metacognitivas & $F(1,182)=109,55 p=, 00$ &, 61 &, 00
\end{tabular}

$\mathrm{R} 2=, 38 \quad \mathrm{R} 2$ Ajustado=,37

Concepción de aprendizaje constructivista

\begin{tabular}{cccc}
\hline Modelo & $F$ & Beta & $p$ \\
\hline Estrategias Metacognitivas & $F(1,182)=139,82 p=, 00$ &, 66 &, 00
\end{tabular}

$\mathrm{R} 2=, 43$ R2Ajustado $=, 43$

Directiva. El modelo seleccionado sobre el tipo de concepción de aprendizaje directiva fue significativo $(F(1,181)=46,05 p=, 00)$. Este modelo explicó el 34\% de la varianza (33\% ajustado) y el nivel de pericia, así como la estrategia metacognitiva de planificación tuvo un efecto significativo sobre la concepción de aprendizaje directivo de los participantes. Sin embargo, el nivel de pericia presenta una mayor peso, $B=-, 63$, $p=, 00$, que la estrategia metacognitiva de aprendizaje, $\mathrm{B}=, 13, \mathrm{p}=, 04$.

Interpretativa. En segundo lugar, el modelo sobre el tipo de concepción interpretativa fue significativo $(F$
$(1,182)=109,55 p=, 00)$. Este modelo explicó el 38\% de la varianza ( $37 \%$ ajustado), en el cual las estrategias metacognitivas de aprendizaje presentaron un efecto significativo sobre la concepción interpretativa de los participantes.

Constructivista. Por último, el modelo sobre el tipo de concepción constructivista fue significativo ( $\mathrm{F}$ $(1,182)=139,82 \mathrm{p}=, 00)$. Este modelo explicó el $43 \%$ de la varianza (43\% ajustado), en el cual las estrategias metacognitivas de aprendizaje presentaron un efecto significativo sobre la concepción constructivista de aprendizaje 


\section{Discusión}

En la presente investigación se analizó tanto el tipo de concepción de aprendizaje que poseen estudiantes de psicología chilenos como el grado de influencia de las variables nivel de pericia y estrategias metacognitivas sobre las concepciones de aprendizaje, reportado por Martínez (2004) en estudiantes de psicología españoles.

En primer lugar, si bien los resultados apuntan a que ambas variables mediadoras se relacionan con la noción de aprendizaje de los estudiantes, en el análisis detallado para cada tipo de concepción se deja en evidencia que para las dimensiones que presentan mayor profundidad, es decir, la interpretativa y constructivista respectivamente, ambas presentan un alto grado de asociación con estrategias metacognitivas. Resultados similares a éstos reportan Vermunt (1998) y Säljö (1979) al determinar la existencia de relaciones significativas entre estudiantes universitarios que poseen concepciones profundas del aprendizaje y el uso de estrategias de autorregulación. En tanto en Chile, Pérez, Valenzuela, Díaz, González-Pienda y Núñez (2013) también encontraron una alta relación entre el uso de estrategias metacognitivas, tales como la planificación y la evaluación, y el grado de disposición al aprendizaje.

En segundo lugar, para el nivel de pericia, los resultados demuestran que aquellos estudiantes que presentaron altas puntuaciones en concepciones de mayor estructuración tendían a encontrarse en un nivel medio o alto de experticia en psicología. Si bien estos resultados se condicen con la evidencia entregada por Alexander et al. (1998) y Boulton-Lewis et al. (2001), a partir del grueso de la información obtenida, no se pudo establecer una distinción precisa y específica para cada tipo de concepción profunda (interpretativa y constructivista) en función de los niveles de dominio específico en psicología.

En tercer lugar, al analizar el grado de influencia de las dos variables predictoras identificadas por Martínez (2004) sobre los tipos de aprendizaje de mayor estructuración, es decir, la concepción interpretativa y constructivista, se evidencia sólo la influencia de la variable de estrategias metacognitivas. Esto quiere decir que si bien existe una relación entre las concepciones de aprendizaje de mayor elaboración y los niveles de pericia más avanzados (quinto y noveno semestre respectivamente), serían las estrategias metacognitivas, las que predicen y diferencian, de mejor manera, el paso de un aprendizaje directivo hacia uno de mayor complejidad y flexibilidad. Este resultado se condice con las investigaciones que señalan que los estudiantes de mayor pericia en algún dominio específico no sólo saben más por el grado de experticia alcanzada, sino también por el conocimiento y uso de estrategias, tales como la planificación, control y evaluación, todas ellas aplicadas al proceso de aprendizaje (Echeverría, Mateos, Pozo \& Scheuer, 2001). Por lo tanto, esta "experticia metacognitiva" influiría significativamente en el dominio específico del área, en este caso la psicología, favoreciendo así procesos de cambio conceptual sobre el aprendizaje mismo y sus contenidos (Nickerson, Perkins \& Smith, 1985). Sin embargo, a modo de limitación de los resultados obtenidos, no se identifican diferencias específicas entre los tipos de concepción interpretativa y constructivista, así como tampoco en el tipo específico de estrategia metacognitiva utilizada. Por lo tanto, posteriores investigaciones debiesen revisar tanto la pertinencia teórica de los constructos a utilizar como la precisión operacional del constructo, reflejada en los instrumentos.

Por otro lado, respecto a la influencia tanto del nivel de pericia como de las estrategias metacognitivas reportadas por Martínez (2004) en estudiantes de psicología españoles, existen diferencias frente a los resultados obtenidos en la presente investigación. Esta identifica, en primer lugar, sólo la influencia de las estrategias metacognitivas sobre las concepciones de mayor profundidad, y, en segundo lugar, la influencia única del nivel de pericia bajo sobre la concepción directiva. A la luz de estas diferencias, posteriores investigaciones debiesen indagar en la identificación de las variables medidadoras de estas diferencias, identificando la posible influencia de variables socioculturales así como de condiciones diferenciales en el proceso de enseñanza aprendizaje disciplinar entre ambos contextos.

En cuarto lugar, respecto a la concepción directiva, sólo para este tipo el nivel de pericia resultó ser la principal variable predictora. Es decir, aquellos estudiantes que presentaron este tipo de concepción tendían a encontrarse cursando el primer semestre de la carrera, así como también, a utilizar escasamente estrategias de aprendizaje autorregulado. A partir de este resultado, a modo de posible explicación, sería interesante indagar en las condiciones de transición de la educación secundaria hacia a la universitaria, con el fin de identificar tanto características como condiciones de continuidad y ruptura en el proceso de enseñanza-aprendizaje. De acuerdo a Pérez et al. (2013), el ingreso al contexto universitario demandaría una mayor exigencia académica, un menor contacto personal entre docente-estudiante y una menor estructuración en las condiciones de enseñanza, en 
contraste con la educación escolar, lo cual influye negativamente sobre la motivación y el aprendizaje. Esta disminución significativa en la motivación se vincularía a aspectos tales como: la cantidad de tareas asignadas, la realización de actividades no significativas para el estudiante, la entrega de evaluaciones sin retroalimentación y la posibilidad de tomar decisiones de manera autónoma, pero sin el apoyo adecuado (Abello, Díaz, Pérez, Almeida, Lagos, González \& Strickland, 2012). Por lo tanto, el ingreso a esta nueva experiencia demanda la capacidad de actuar con mayor autorregulación, lo que conlleva aprender a compatibilizar las exigencias académicas de la carrera universitaria con las nuevas condiciones de autonomía (Pérez et al., 2013).

Por último, a la luz de los antecedentes y resultados presentados con respecto a la metacognición, el nivel de pericia y las concepciones de aprendizaje, posteriores investigaciones aplicadas al contexto universitario debiesen indagar en las posibles relaciones e influencia en ámbitos aplicados tales como el diseño curricular, la didáctica de la enseñanza y la evaluación del aprendizaje. Adicionalmente, se debería de explorar, como posible aporte, la comprensión de las altas tasas de fracaso y deserción universitaria en el primer año, complementando así, la influencia del factor socioeconómico con variables mediacionales que pudiesen influir en el desempeño académico (Centro de Microdatos, 2008). De acuerdo a este último antecedente, algunos estudios reportan que aquellos estudiantes que presentan un desempeño exitoso, tienden a poseer y utilizar la capacidad de autorregulación cognitiva, la que junto a sus habilidades académicas y personales posibilitan el control y ajuste de las conductas de aprendizaje (Rosário, Núñez, González-Pienda, Almeida, Soares \& Rubio, 2005). A su vez, para Ruban y Reis (2006) existiría una estrecha relación entre la utilización de estrategias de autorregulación del aprendizaje, el enfoque de aprendizaje profundo y las metas de aprendizaje académico.

A modo de cierre y bajo el marco de la problemática educativa presentada, surgen las siguientes interrogantes: ¿Qué pasaría si estudiantes, previo ingreso a la universidad o paralelo a su formación profesional, pudiesen adquirir y desarrollar estrategias metacognitivas de aprendizaje? ¿Podría favorecer la adquisición y desarrollo de estrategias metacognitivas la profundización de los procesos y contenidos del aprendizaje en dominios específicos del conocimiento? (Hernández Pina, 2002). Las respuestas a estas interrogantes debiesen ser exploradas por futuras investigaciones, aplicables no sólo en el contexto universitario, como es el caso, sino a todo campo investigativo que se preocupe y ocupe del estudio del cambio conceptual y del aprendizaje en general.

\section{Referencias}

Abello, R., Díaz, A., Pérez, M., Almeida, L., Lagos, I., González, J. \& Strickland B. (2012). Vivencias e implicación académica en estudiantes universitarios: adaptación y validación de escalas para su evaluación. Estudios Pedagógicos, 38(2), 7-19. doi:10.4067/ S0718-07052012000200001

Alexander, P., Murphy, K., Guan, J., \& Murphy, P. (1998). How students and teachers in Singapore and the United States conceptualize knowledge and beliefs: Positioning learning within epistemological frameworks. Learning and Instruction, 8(2), 97-116. doi:10.1016/S0959-4752(97)00004-2

Boekaerts, M. (1999). Self-regulated learning: Where we are today. International journal of educational research, 31(6), 445-457. doi:10.1016/S0883-0355(99)00014-2

Boekaerts, M. \& Niemivirta, M. (2000). Self-regulated learning: Finding a balance between learning goals and ego-protective goals. En M. Boekaerts, P. Pintrich \& M. Zeidner (Eds.), Handbook of self-regulation: Theory, research, and applications (pp. 417-450). San Diego, CA: Academic Press.

Boulton-Lewis, G., Marton, F., Lewis, D. \& Wilss, L. (2000). Aboriginal and Torres Strait Islander university students' conceptions of formal learning and experiences of informal learning. Higher Education, 39(4), 469-488. doi:10.1023/A:1004060422023

Boulton-Lewis, G., Smith, D., McCrindle, A., Burnett, P. \& Campbell, K. (2001). Secondary teachers' conceptions of teaching and learning. Learning and instruction, 11(1), 35-51. doi:10.1016/ S0959-4752(00)00014-1

Brew, A. (2001). Conceptions of research: A phenomenographic study. Studies in higher education, 26(3), 271-285. doi:10.1080/03075070120076255

Brown, A., Bransford, J., Ferrara, R. \& Campione, J. (1983). "Learning, remembering, and understanding" En J. Flavell y E. Markman (Eds.), Handbook of child psychology: Vol.3. Child development (pp. 77-166). New York: Wiley.

Carey, S. (1985a). Conceptual Change in Childhood. Cambridge, MA: Bradford Books, MIT Press.

Carey, S. (1985b). Are children fundamentally different thinkers and learners from adults? En S. Chipman, J. Segal \& R. Glaser (Eds.), Thinking and Learning Skills, 2. (pp. 485-517). Hillsdale, NJ: Erlbaum. Reprinted by Open University Press: Open University Readings in Cognitive Development.

Centro de Microdatos (2008). Informe Final: Estudios sobre causas de la deserción universitaria. Departamento de Economía, Universidad de Chile. Recuperado de http://www.opech.cl/educsuperior/ politicaacceso/informefinalcausasdesercionuniversitaria.pdf

Danserau, D. (1978). The development of a learning strategy curriculum. En H. O'Neil (Ed.), Learning strategies (pp. 1-29). New York: Academic Press.

DeCharms, R. (1972). Personal causation training in the schools. Journal of Applied Social Psychology, 2(2), 95-113. doi:10.1111/j.1559-1816.1972.tb01266.x

Dweck, C. (1975). The role of expectations and attributions in the alleviation of learned helplessness. Journal of personality and social psychology, 31(4), 674-684. doi:10.1037/h0077149 
Dykstra, D., Boyle, F. \& Monarch, I. (1992). Studying conceptual change in learning physics. Science Education, 76(6), 615-652. doi:10.1002/sce. 3730760605

Echeverría, M., Mateos, M., Pozo, J. \& Scheuer, N. (2001). En busca del constructivismo perdido: concepciones implícitas sobre el aprendizaje. Estudios de psicología, 22(2), 155-173. doi:10.1174/021093901609479

Flavell, J. (1979). Metacognition and cognitive monitoring: A new area of cognitive-developmental inquiry. American psychologist, 34(10), 906-911. doi:10.1037/0003066X.34.10.906

Glaser, R. (1982). Instructional psychology: Past, present, and future. American Psychologist, 37(3), 292-305. doi:10.1037/0003066X.37.3.292

Hernández Pina, F. (2002). Docencia e investigación en educación superior. Revista de investigación educativa, 20(2), 271-301. Recuperado de http://revistas.um.es/rie/article/view/98921

Hewson, P. \& Thorley, N. (1989). The conditions of conceptual change in the classroom. International Journal of Science Education, 11(5), 541-553. doi:10.1080/0950069890110506

Kuhn, D. \& Lao, J. (1998). Contemplation and conceptual change: Integrating perspectives from social and cognitive psychology. $D e-$ velopmental Review, 18(2), 125-154. doi:10.1006/drev.1997.0449

Limón, M. (2001). On the cognitive conflict as an instructional strategy for conceptual change: a critical appraisal. Learning and instruction, 11(4), 357-380. doi:10.1016/S0959-4752(00)00037-2

Lonka, K., Joram, E. \& Bryson, M. (1996). Conceptions of learning and knowledge: Does training make a difference?. Contemporary Educational Psychology, 21(3), 240-260. doi:10.1006/ ceps.1996.0021

Máiquez, M., Rodrigo, M., Capote, C. \& Vermaes, I. (2000). Aprender en la vida cotidiana: un programa experiencial para padres. Madrid: Visor

Marshall, D., Summer, M. \& Woolnough, B. (1999). Students' conceptions of learning in an engineering context. Higher education, 38(3), 291-309. doi:10.1023/A:1003866607873

Martínez, J. (1999). Aprendizaje en la Universidad: desafíos para el siglo XXI. Del énfasis en los productos al énfasis en los procesos. Número Monográfico: Aprendizaje. Revista Latinoamericana de Psicología, 31(3), 491-504. Recuperado de http://www.redalyc. org/articulo.oa?id=80531305

Martínez, J. (2000). Motivación, estrategias de aprendizaje y evaluación del rendimiento en alumnos universitarios. Iberpsicologia: Revista Electrónica de la Federación española de Asociaciones de Psicología, 5(2), 2. Recuperado de http://dialnet.unirioja.es/ servlet $/$ articulo? codigo $=300458$.

Martínez, J. (2004). Concepción de aprendizaje, metacognición y cambio conceptual en estudiantes universitarios de psicología (Tesis Doctoral, Universitat de Barcelona). Recuperada de http:// diposit.ub.edu/dspace/handle/2445/42698/.

Mischel, W. \& Baker, N. (1975). Cognitive appraisals and transformations in delay behavior. Journal of Personality and Social Psychology, 31(2), 254 - 261. doi:10.1037/h0076272

Nickerson, R., Perkins, D. \& Smith, E. (1985). The teaching of thinking. Hillsdale, NJ: Lawrence Erlbaum Associates.

Nisbet, J. y Shucksmith, J. (1987). Estrategias de Aprendizaje. Madrid: Santillana.

O'Neil, H. \& Abedi, J. (1996). Reliability and validity of a state metacognitive inventory: Potential for alternative assessment. The Journal of Educational Research, 89(4), 234-245. doi:10.1080/0 0220671.1996 .9941208

Pérez, M., Valenzuela, M., Díaz, A., González-Pienda, J. \& Núñez, J. (2013). Dificultades de aprendizaje en estudiantes universitarios de primer año. Atenea, (508), 135-150. doi: 10.4067/S071804622013000200010

Pintrich, R., Marx, R. \& Boyle, R. (1993). Beyond cold conceptual change: The role of motivational beliefs and classroom contextual factors in the process of conceptual change. Review of Educational research, 63(2), 167-199. doi:10.3102/00346543063002167

Pozo, J. (2003). Adquisición de conocimiento: cuando la carne se hace verbo. Madrid: Morata.

Pozo, J. \& Scheuer, N. (1999). Las concepciones sobre el aprendizaje como teorías implícitas. En J. Pozo y C. Monereo (Eds.), El aprendizaje estratégico (pp. 87-108). Madrid: Santillana.

Pozo, J. \& De La Cruz, M. (2003). Concepciones sobre el currículo universitario ¿centrado en los contenidos o centrado en los alumnos? En C. Monereo y J. Pozo (Eds.), La universidad ante la nueva cultura educativa: enseñar y aprender para la autonomía (pp. 141-154). Madrid: Síntesis.

Reif, F. \& Larkin, J. (1991). Cognition in scientific and everyday domains: Comparison and learning implications. Journal of Research in Science Teaching, 28(9), 733-760. doi:10.1002/tea.3660280904

Rodríguez Moneo, M. \& Carretero, M. (1996). Adquisición de conocimiento y cambio conceptual. Implicaciones para la enseñanza de las ciencias. En M. Carretero (Ed.), Construir y Enseñar: Las Ciencias Experimentales (pp. 47-73). Buenos Aires: Aique.

Rosário, P., Núñez, J., González-Pienda, J., Almeida, L., Soares, S. \& Rubio, M. (2005). El aprendizaje escolar examinado desde la perspectiva del 'Modelo 3P' de J. Biggs. Psicothema, 17(1), 20-30. Recuperado de http://www.psicothema.com/psicothema. asp?id=3059

Ruban, L. \& Reis, S. (2006). Patterns of self-regulatory strategy among low-achieving and high achieving university students. Roeper Review, 28(3), 148-156. doi:10.1080/02783190609554354

Säljö, R. (1979). Learning in the learners' perspective. I. Some common sense conceptions. Reports from the Institute of Education, University of Göteborg, (76), 656-658. Recuperado http://eric. ed.gov/?id=ED173369

Schommer, M. (1993). Epistemological development and academic performance among secondary students. Journal of Educational Psychology, 85(3), 406-411. doi:10.1037/0022-0663.85.3.406

Schommer, M., Calvert, C., Gariglietti, G. \& Bajaj, A. (1997). The development of epistemological beliefs among secondary students: A longitudinal study. Journal of Educational Psychology, 89(1), 37-40. doi:10.1037/0022-0663.89.1.37

Schraw, G. \& Moshman, D. (1995). Metacognitive theories. Educational psychology review, 7(4), 351-371. doi:10.1007/BF02212307

Tynjälä, P. (1997). Developing education students' conceptions of the learning process in different learning environments. Learning and Instruction, 7(3), 277-292. doi:10.1016/S0959-4752(96)00029-1

Tynjälä, P. (1999). Towards expert knowledge? A comparison between a constructivist and a traditional learning environment in the university. International journal of educational research, 31(5), 357-442. doi:10.1016/S0883-0355(99)00012-9

Valle, A., González, R., Núñez, J., Vieiro, P., Gómez, M. \& Rodríguez, S. (1999). Un modelo cognitivo-motivacional explicativo del rendimiento académico en la universidad. Estudios de psicología, 20(62), 77-100. doi:10.1174/02109390260288631

Vermunt, J. (1996). Metacognitive, cognitive and affective aspects of learning styles and strategies: A phenomenographic analysis. Higher education, 31(1), 25-50. doi:10.1007/BF00129106

Vermunt, J. (1998). The regulation of constructive learning processes. British journal of educational psychology, 68(2), 149-171. doi:10.1111/j.2044-8279.1998.tb01281.x

Villalobos, E., Guerrero, M., Pérez, R., Avendaño, A., Ceballos, A., Ortiz, J. \& Parra, C. (2009). Estilos de aprendizaje y metodologías de 
EN ESTUDIANTES UNIVERSITARIOS DE PSICOLOGÍA

enseñanza en estudiantes de obstetricia. Educación médica, 12(1), 4346. Recuperado de http://scielo.isciii.es/pdf/edu/v12n1/original3.pdf
White, R. \& Gunstone, R. (1989). Metalearning and conceptual change. International Journal of Science Education, 11(5), 577586. doi:10.1080/0950069890110509 
